\title{
INVESTIGAÇÃO DO USO DE DROGAS LÍCITAS E ILÍCITAS ENTRE OS UNIVERSITÁRIOS DE INSTITUIÇÕES DO ENSINO SUPERIOR (PÚBLICAS E PRIVADAS), no município de Picos, Piauí
}

\author{
Rivelilson Mendes de Freitas ${ }^{1}$ \\ Danelle da Silva Nascimento ${ }^{2}$ \\ Pauline Sousa dos Santos ${ }^{3}$
}

\begin{abstract}
O trabalho investigou a prevalência do uso de substâncias psicoativas lícitas e ilícitas entre os universitários das instituições de ensino superior (públicas e privadas) do município de Picos, PI. O estudo foi realizado por meio de um questionário elaborado de forma estruturado e autoaplicado entre 3.600 universitários. O perfil dos universitários é de mulheres adultas $(61,5 \%)$, brancas $(41,6 \%)$, solteiras $(80,4 \%)$, faixa etária entre 17 e 22 anos $(55,6 \%)$ e que não exerciam atividade remunerada. Dentre os estudantes, $74,5 \%$ não praticava automedicação e nem fazia uso de psicotrópicos, no entanto, em eventos sociais, fazia uso de álcool $(61,2 \%)$ e de tabaco $(18,5 \%)$, principalmente pela influência dos amigos $(27,3 \%)$.
\end{abstract}

Descritores: Epidemiologia; Alcoolismo; Drogas Ilícitas; Estudantes.

\footnotetext{
${ }^{1}$ PhD, Professor Adjunto, Universidade Federal do Piauí, Teresina, PI, Brasil.

2 Enfermeira, Professor Substituto, Fundação Universidade Estadual do Piauí, Teresina, PI, Brasil.

${ }^{3}$ Aluna do curso de graduação em farmácia, Universidade Federal do Piauí, Teresina, PI, Brasil.
} 


\section{INVESTIGATION OF LICIT AND ILLICIT DRUG USE AMONG COLLEGE STUDENTS (From PUblic AND PRIVATe institutions) in the Municipality of Picos, Piauí}

This study has investigated the prevalence of licit and illicit psychoactive substances among college students of Higher Education Institutions (IES) (public and private) of the municipality of Picos. It was conducted through a self-administered questionnaire with 3,600 college students. The students' profile is composed of adult women (61.5\%); white (41.6\%); single (80.4\%); age group from 17 to 22 years old $(55.6 \%)$ who do not have their own income. Among the students, $74.5 \%$ do not practice self-medication nor use of psychotropic drugs, however, in social events they drink alcohol (61.2\%) and smoke cigarettes (18.5\%), mainly because the influence of friends $(27.3 \%)$.

Descriptors: Epidemiology; Alcoholism; Drugs; Students.

\section{AVERIguACIÓN DEL USO DE DROGAS LÍCITAS Y ILÍCITAS ENTRE LOS UNIVERSITARIOS DE INSTITUCIONES DE LA ENSEÑANZA SUPERIORA (PÚBLICAS Y privadas) en el municipio de Picos, Piauí}

El trabajo investigó la superioridad del uso de substancias psicoactivas lícitas e ilícitas entre los universitarios de las instituciones de enseñanza superiora (IES) (públicas y privadas) del municipio de Picos. El estudio fue realizado por medio de un cuestionario elaborado de forma estructurado y auto-aplicado entre 3.600 universitarios. El perfil de los universitarios es de mujeres adultas $(61,5 \%)$, blancas $(41,6 \%)$, solteras $(80,4 \%)$, con banda etaria entre 17 y 22 años $(55,6 \%)$ que no ejercen actividad remunerada. Entre los estudiantes, $74,5 \%$ no practica automedicación y ni hace uso de psicotrópicos, sin embargo, en eventos sociales hace uso del alcohol $(61,2 \%)$ y del tabaco $(18,5 \%)$, principalmente por la influencia de los amigos (27,3\%).

Descriptores: Epidemiología; Alcoholismo; Drogas ilícitas; Estudiantes.

\section{Introdução}

O abuso de drogas caracteriza-se como fenômeno disseminado em todo o mundo com consequências graves. O consumo dessas substâncias prejudiciais ao organismo humano é particularmente preocupante na população jovem, devido aos altos custos sociais. No Brasil, jovens entre 18 e 24 anos, especialmente estudantes universitários, apresentam altas prevalências na vida no consumo, tanto para drogas lícitas quanto para as ilícitas ${ }^{(1)}$. A precoce relação entre as drogas e o usuário pode ser constatada em diversos trabalhos registrados na literatura. Entre os vários fatores que apontam para o crescimento da distribuição e do consumo no país, encontra-se o quadro psicossocial do jovem que busca fugir da realidade difícil, ou pelo prazer rápido e fácil obtido pelo uso de $\operatorname{drogas}^{(2)}$.
$O$ ingresso na universidade, ainda que traga sentimentos positivos e de alcance de uma meta programada pelos estudantes do ensino médio, por vezes pode se tornar um período crítico, de maior vulnerabilidade para o início e a manutenção do uso de álcool e de outras drogas ilícitas ${ }^{(2)}$. Os fatores relacionados ao processo de iniciação dos jovens, em relação ao consumo de drogas, são de interesse crescente, uma vez que a elucidação do processo pelo qual essa população fica exposta ao consumo de substâncias pode propiciar o desenvolvimento de estratégias mais eficazes de prevenção e de tratamento ${ }^{(2)}$.

Segundo o I Levantamento Nacional sobre Uso de Álcool, Tabaco e outras Drogas entre Universitários, realizado pela Secretaria Nacional de Políticas sobre 
Drogas (Senad), em parceria com o Grupo Interdisciplinar de Estudos de Álcool e Drogas da Faculdade de Medicina da Universidade de São Paulo, realizado em 2009, com 18.000 universitários das 27 capitais federais do Brasil, constatou-se que o problema de abuso de drogas é algo alarmante dentro da nossa sociedade, já que os estudos mostraram que $49 \%$ dos universitários pesquisados experimentaram alguma droga ilícita pelo menos uma vez na vida; e, no grupo dos universitários que se declararam menores de 18 anos, 80\% dos entrevistados afirmaram já ter consumido algum tipo de bebida alcoólica ${ }^{(3)}$.

Além disso, esse mesmo estudo mostrou que $86 \%$ dos universitários já fizeram uso de álcool e $47 \%$ de produtos derivados do tabaco, $22 \%$ dos universitários apresentaram risco de desenvolver dependência ao álcool e $8 \%$ à maconha e, aproximadamente, 40\% dos universitários usaram duas ou mais drogas nos últimos 12 meses e $43 \%$ relataram já ter feito uso múltiplo e simultâneo de drogas na vida. A prevalência de abuso de álcool foi maior entre os universitários do que na população em geral, entretanto, a dependência química apresentou maior prevalência entre os usuários dessa população ${ }^{(3)}$. Esses fatos mostram que se faz necessária a implantação de campanhas de conscientização desses jovens e futuros profissionais em relação à prevenção e combate ao uso dessas substâncias e sobre as suas consequências psicossociais.

A literatura demonstra que algumas das consequências do consumo de drogas entre estudantes universitários são acidentes automobilísticos, violência familiar, comportamento sexual de risco, prejuízos no processo de ensino e aprendizagem, diminuição da percepção e estresse ${ }^{(4-7)}$. Outro estudo também destaca que há diminuição da expectativa de vida, uma vez que os comportamentos de risco associados ao consumo de álcool e drogas podem afetar o senso global de "bem-estar"(8).

Considerando a alta prevalência do uso de drogas entre estudantes universitários e a carência de estudos, especificamente na Região Nordeste, sobre esse problema de saúde pública, o estudo foi conduzido com os seguintes objetivos: realizar um levantamento sobre uso de drogas entre os universitários do município de Picos, Piauí, avaliar os fatores de risco relacionados ao uso indevido de drogas entre os universitários da população em estudo, determinar o padrão de consumo de drogas entre os universitários, verificar o grau de associação entre a situação socioeconômica e o uso de álcool, tabaco, psicofármacos e "drogas ilícitas" entre universitários, identificar os principais medicamentos usados na prática da automedicação e o grau de orientação entre os universitários sobre o uso de drogas e as suas consequências.

\section{Material e Métodos}

\section{Desenho do estudo}

Os resultados foram obtidos por meio de um estudo do tipo exploratório descritivo, com abordagem quantitativa $^{(9-10)}$, por meio de uma entrevista realizada diretamente com os universitários, de forma aleatória, durante aproximadamente 20 minutos antes das suas atividades acadêmicas. Durante as entrevistas com os universitários $(n=3.600)$ regularmente matriculados em um dos cursos de graduação ofertados por todas as instituições $(n=3)$ de ensino superior do município de Picos, Piauí, foi autoaplicado um questionário previamente elaborado com perguntas claras, objetivas e de múltiplas respostas. Esse questionário foi elaborado pelo coordenador do grupo, baseado em dados disponíveis de outros estudos sobre a temática em questão ${ }^{(4-6)}$. Foram incluídos no estudo os universitários regularmente matriculados e aqueles que se encontravam em atividades escolares frequentes em todas as instituições de ensino superior do município de Picos, Piauí. As entrevistas foram realizadas durante o período de abril a novembro de 2009. Os universitários das três instituições que não quiseram participar do projeto, no momento da aplicação dos questionários pelo grupo de pesquisa, não foram entrevistados $(n=406)$.

$\mathrm{O}$ protocolo de pesquisa cumpriu os princípios éticos contidos na Declaração de Helsinki e as normas da Resolução 196/96, do Conselho Nacional de Saúde, tendo sido aprovado pelo Comitê de Ética em Pesquisa da Universidade Federal do Piauí (CAAE: 0086.0.045.00009).

\section{Locais do estudo}

Os locais de estudo foram todas as Instituições de Ensino Superior (IES) do município de Picos, Piauí, descritas a seguir: Universidade Federal do Piauí (UFPI), Universidade Estadual do Piauí (UESPI) e Faculdade R. Sá. Foram utilizados como amostra de conveniência entre os estudantes universitários do município de Picos, 3.600 alunos das três IESs participantes do projeto do $1^{\circ}$ ao $9^{\circ}$ período acadêmico, sendo estudantes dos seguintes cursos de graduação ofertados pela UFPI (1.200 acadêmicos) - administração (335), ciências biológicas (246), enfermagem (267), letras (235) e pedagogia (117); pela UESPI (1.200 acadêmicos) - administração (137), ciências biológicas (137), ciências contábeis (154), direito (256), enfermagem (152), sistemas de informação (102) e letras (262) e pela Faculdade R. Sá (1.200 acadêmicos) - administração (292, ciências contábeis (218), direito (227), sistemas de informação (248) e pedagogia (215).

Selecionou-se como variável independente: o hábito de consumir drogas lícitas e ilícitas e, como variáveis dependentes, o início do hábito do consumo, o motivo que desencadeou esse hábito, e, no caso do tabaco, foi verificada a quantidade de cigarros fumados por dia e o tipo de fumo utilizado. O padrão de uso durante a vida foi definido no estudo como o hábito de consumir drogas lícitas e ilícitas pelo menos uma vez na vida.

\section{Coleta dos dados}

Os dados foram coletados de abril a novembro de 2009. A coleta dos dados foi realizada pelos mesmos acadêmicos do Curso de Enfermagem da Universidade Federal do Piauí, treinados e sob supervisão. Os registros dos dados dos sujeitos foram identificados pelo curso/ instituição. Esses registros foram feitos em questionários 
próprios específicos, baseados em estudos anteriores e adaptados para a realidade da região, também para que esse possa ser utilizado como base para novos estudos, tanto no próprio no município como nos demais municípios do Estado $^{(8-10)}$. O questionário foi dividido em quatro partes, sendo essas: dados socioeconômicos, dados relacionados ao consumo de substâncias lícitas ou ilícitas, dados em relação ao consumo de tabaco e grau de informação. A partir das perguntas de cada sessão do questionário, obtiveram-se as seguintes informações: idade, sexo, cor da pele autorreferida, estado civil, número de filhos, renda familiar, curso de graduação, tipo de moradia, uso de medicação com potencial de abuso, uso de drogas lícitas e licitas e informações relacionados à frequência do uso de drogas. Todos os universitários convidados a participar foram esclarecidos quanto ao objetivo da entrevista e, quando aceitaram participar, sempre de forma voluntária, assinaram o Termo de Consentimento Livre e Esclarecido (TCLE).

Para o tratamento dos dados, foi produzido um banco de dados no programa Microsoft Excel e utilizado o programa estatístico SPSS, versão 15.0. Para análise e interpretação dos resultados, para as três instituições de ensino superior investigadas, foram usados os resultados existentes na literatura e nos estudos encontrados nas bases de dados MEDLINE, LILACS, SciELO e Adolec, durante as consultas realizadas no mês de janeiro de 2010. A estatística descritiva com emprego da frequência absoluta e percentual foi utilizada para análise das informações. Os resultados foram considerados significativos quando $\mathrm{p}<0,05$.

\section{Resultados}

As instituições de ensino superior do município de Picos estudadas são referentes ao ensino público e privado do Estado do Piauí. Assim, esses resultados têm limitações, mas são representativos quanto aos universitários do Estado do Piauí. Entre os universitários entrevistados foi vista uma frequência de distribuição dos mesmos nos seguintes cursos de graduação: administração $(21,2 \%)$, letras $(13,8 \%)$, direito $(13,4 \%)$, enfermagem $(11,6 \%)$, ciências biológicas $(10,6 \%)$, ciências contábeis $(10,3 \%)$, ciências da computação $(9,7 \%)$ e pedagogia $(9,4 \%)$.

Dos 3.600 universitários acompanhados no estudo, a maioria foi composta pelo gênero feminino $(61,5 \%)$, $55,6 \%$ tinham idade entre 17 e 22 anos (Figura 1), 41,6\% autorreferiu a cor da pele como branca e 36,3\% parda. Quanto à situação conjugal, 80,4\% deles eram solteiros e $51,6 \%$ apresentam renda familiar de dois a quatro saláriosmínimos (Figura 2).

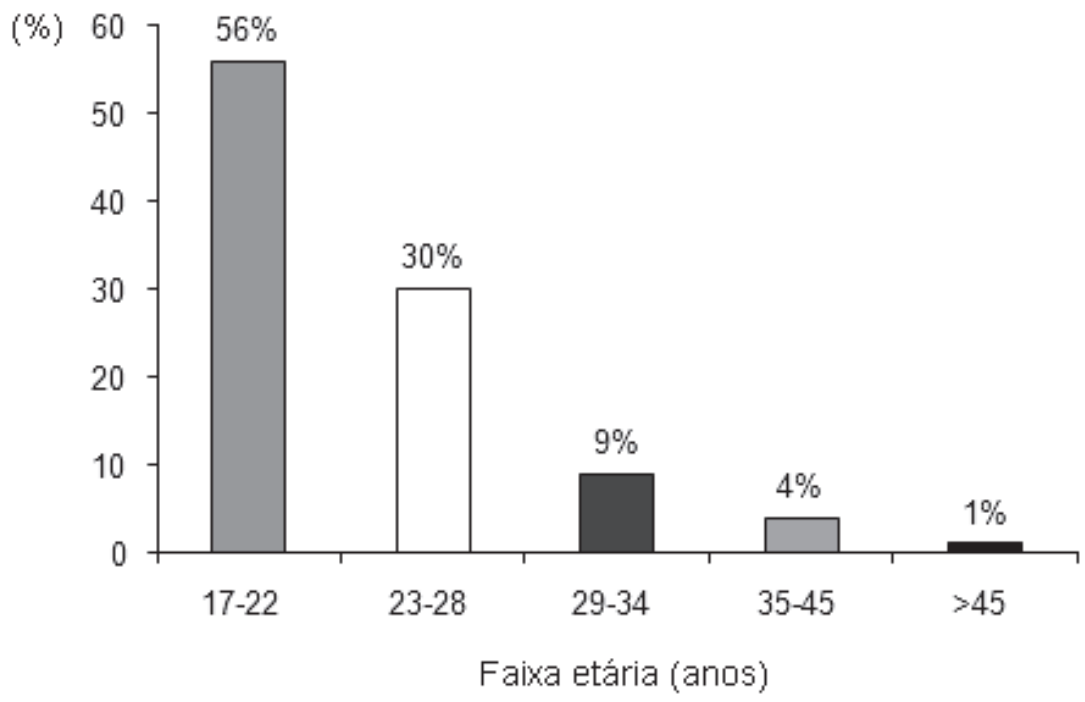

Figura 1 - Distribuição dos universitários das instituições de ensino superior do município de Picos, segundo a faixa etária em anos. Picos, PI, Brasil 


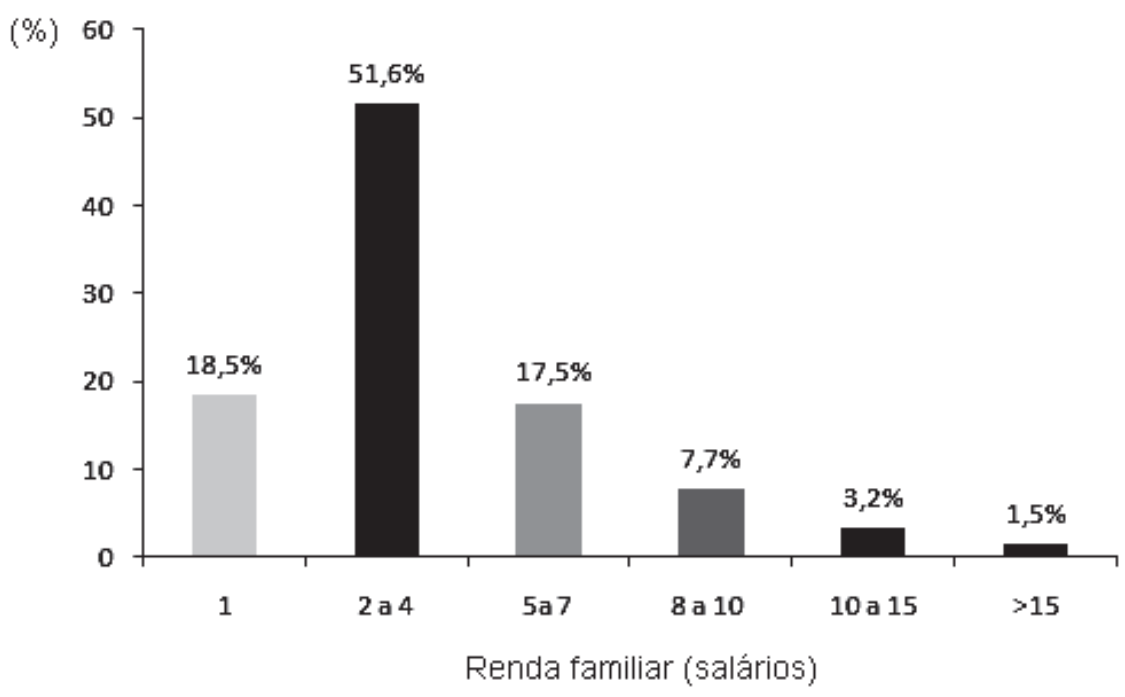

Figura 2 - Distribuição dos universitários das instituições de ensino superior do município de Picos, segundo a renda familiar em salários-mínimos. Picos, PI, Brasil

Quanto às possíveis drogas utilizadas entre os universitários, foi detectada alta prevalência de consumo para as drogas lícitas como o álcool $(61,2 \%)$ e o tabaco $(18,5 \%)$, entre os universitários das três instituições de ensino superior do município de Picos. Também foi visto que $27,3 \%$ faziam uso dessas substancias durante eventos sociais e, principalmente, devido à influência de amigos. Além disso, foi verificada alta frequência para o uso da maconha $(7,9 \%)$ e de substâncias tranquilizantes $(6,7 \%)$ (Figura 3).

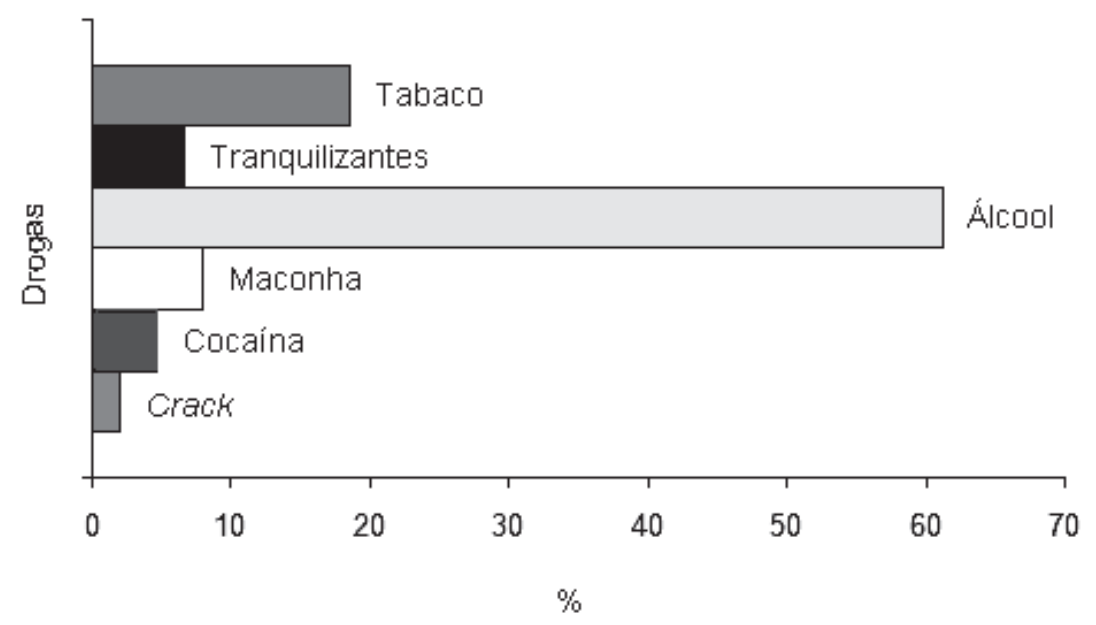

Figura 3 - Distribuição das principais substâncias lícitas e ilícitas usadas entre os universitários das instituições de ensino superior do município de Picos. Picos, PI, Brasil

Os estudantes destacaram a influência dos amigos $(27,3 \%)$ e a sensação de diversão $(22,8 \%)$ causada pelas drogas como as principais motivações para o uso dessas substâncias (Figura 4), sendo que a grande maioria relatou não usar o álcool de modo frequente e não haver se excedido no seu uso $(70,5 \%)$. 


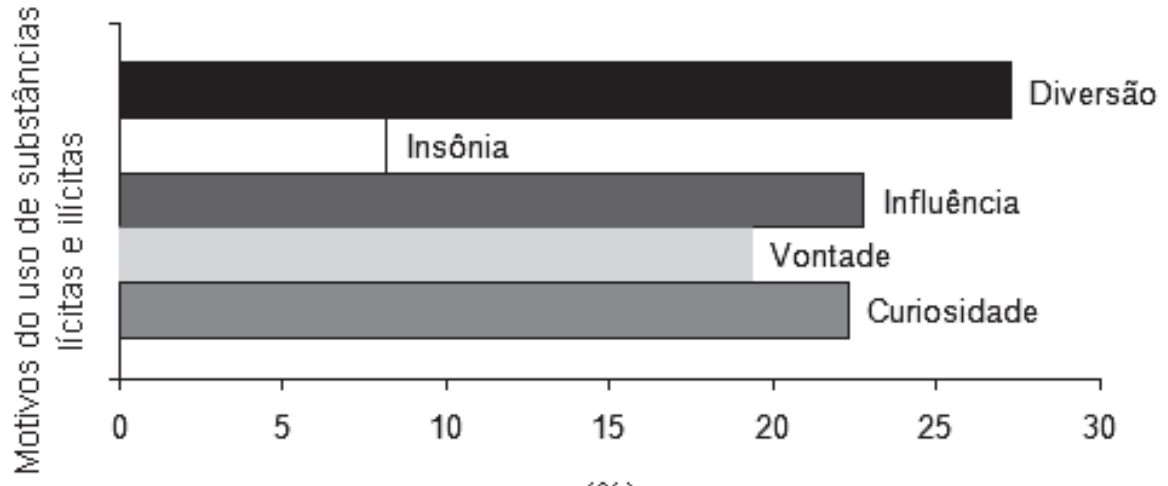

$(\%)$

Figura 4 - Distribuição dos principais motivos que induziram ao uso de substâncias lícitas e ilícitas entre os universitários das instituições de ensino superior do município de Picos. Picos, PI, Brasil

Em relação à prática da automedicação, os resultados deste estudo demonstram que 74,5\% dos universitários não faziam uso de medicamentos sem prescrição médica. No entanto, $25,5 \%$ deles relataram fazer uso de analgésicos e anti-inflamatórios sem prescrição médica.

\section{Discussão}

Os resultados, aqui, para o perfil socioeconômico entre os universitários, corroboram os de outros estudos que envolvem o uso de drogas lícitas e ilícitas entre universitários. Em estudos anteriores, foram encontrados resultados semelhantes aos aqui encontrados, para os estudantes do município de Picos, Piauí, para as seguintes variáveis: sexo, faixa etária, cor da pele autorreferida, situação conjugal e renda familiar, entre os universitários acompanhados ${ }^{(11-12)}$.

Com relação ao sexo feminino, à cor da pele autorreferida, à situação conjugal e à renda familiar, os dados detectados corroboram os de outros estudos que envolvem o uso de drogas lícitas e ilícitas entre universitários. Em estudos anteriores, foram encontrados resultados semelhantes para as mesmas variáveis avaliadas entre os universitários acompanhados ${ }^{(11-12)}$. Quanto às possíveis drogas utilizadas entre os universitários, os achados desta pesquisa estão de acordo com outros trabalhos encontrados na literatura ${ }^{(11-13)}$.

Segundo um estudo desenvolvido entre estudantes da área da saúde de uma universidade de Curitiba, Paraná, o consumo de álcool ou drogas, entre os participantes que já fizeram uso, era induzido principalmente pelos seus "colegas, amigos ou conhecidos"(14). Esse estudo demonstrou que $65,8 \%$ dos estudantes nos quatro cursos estudados fizeram uso, pelo menos uma vez, dessas substâncias. Quanto ao principal motivo de uso que induziu ao uso pela primeira vez, $13,6 \%$ dos alunos do curso de educação física informaram que começaram a usar em busca de "diversão ou prazer". Entretanto, 6,4\% dos universitários relataram que fizeram uso dessas substâncias pela primeira vez para "melhorar o desempenho" (escolar, sexual e/ou social). Outros motivos que induziram ao uso dessas substâncias pela primeira vez foram a curiosidade $(18,7 \%)$ e a busca pela diversão ou prazer fácil $(14,1 \%)^{(14)}$.

Sobre esses fatores indutores ao consumo de drogas licítas e ilícitas, pode ser verificado que o ambiente universitário se torna favorável para o uso de bebidas alcoólicas e de outras drogas, uma vez que há diversos eventos sociais que favorecem e torna atrativo o uso do álcool e de outras drogas ilícitas ${ }^{(15)}$.

Estudo realizado com acadêmicos do curso de enfermagem, em uma universidade de Passo Fundo, $\mathrm{RS}$, revelou que bares, danceterias, boates $(31,5 \%)$ e a casa de amigos/conhecidos (18,2\%) são os lugares mais propícios para o consumo de drogas e em especial o álcool, sendo que amigos e familiares foram citados como os principais responsáveis pela introdução do acadêmico no uso de bebidas alcoólicas, numa frequência de 49 e $20 \%$, respectivamente ${ }^{(16)}$.

Quanto ao uso do cigarro entre os universitários investigados nas três instituições de ensino superior do município de Picos, 58,9\% se autorreferiu como não fumante, revelando, ainda, não haver qualquer influência pelo hábito do fumo por parte dos seus familiares, uma vez que $72,9 \%$ dos pais dos universitários não fumam e apenas $18 \%$ deles o pai ou mãe faziam uso de tabaco. Novamente destaca-se a influência dos amigos para o início de uma atividade prejudicial à saúde. Os estudantes entrevistados afirmaram que o começo do hábito de fumar deve-se, principalmente, à influência de amigos que já faziam uso do tabaco. No presente estudo detectou-se que 40,3\% fizeram uso do tabaco devido à influência de amigos, também foi verificado no estudo que 20,5\% fizeram o uso dessa substância pelo sentimento de independência conseguido com o uso do cigarro ${ }^{(16)}$.

O ingresso dos adolescentes no ambiente universitário constitui importante passo para a vida profissional. É uma inegável oportunidade para o desenvolvimento profissional, social e familiar, no entanto, também é uma fase que pode ser relacionada a diversos fatores de risco. Dentre eles o consumo de substâncias e as suas consequências, bem 
como o fácil acesso às substâncias ilícitas, propiciadas pelo narcotráfico, que comercializa essas substâncias nos arredores das universidades, bem como associado ao uso dessas em eventos sociais.

Entre as três instituições de ensino superior do município de Picos acompanhadas no estudo, pode ser verificado alto grau de consumo de substâncias psicoativas, em especial o álcool, devido, principalmente, à influência de amigos em eventos sociais, constituindo, assim, um local de contraste social e de algumas práticas indevidas em relação ao consumo dessas substâncias. O uso dessas substâncias pode trazer consequências, prejuízos psicossociais e até ocasionar morte entre os universitários $^{(17)}$. É possível inferir que o consumo de drogas de abuso tem causado considerável interferência nas atividades acadêmicas dos alunos e que representa alto risco de dependência química, bem como pode proporcionar maior incidência de morbidades psiquiátricas e comprometimento da atuação profissional.

Além disso, os achados, aqui, também revelam que os universitários possuem conhecimentos e são esclarecidos sobre as consequências para a prática da automedicação, uma vez que durante a mesma há alto número de erros de administração durante a escolha da dose, da via e da posologia.

Pode-se, assim, sugerir que exista influência do ambiente universitário para o consumo abusivo de substâncias psicoativas, o que confere maior importância à tomada de medidas preventivas institucionais. A prevenção do uso abusivo de drogas é essencial para evitar danos à saúde e à qualidade de vida dos universitários, bem como para reduzir uma posterior dependência química. Os resultados obtidos ressaltam a importância da triagem do uso de substâncias nas instituições de ensino em questão para inserção de programas de prevenção direcionados à epidemiologia local.

\section{Considerações Finais}

Este estudo, com abordagem quantitativa, foi resultado da participação direta nas atividades acadêmicas dos universitários de instituições de ensino superior do município de Picos, Piauí. Considerando tratar-se de IESs de referência para o ensino universitário, houve interesse em traçar o perfil dos universitários assistidos, acreditandose que se poderia, então, reconhecer suas características e identificar fatores de risco associados ao uso de drogas lícitas e ilícitas para que no futuro possam ser adotadas intervenções educativas para combater e prevenir o uso de drogas.

Programas de prevenção do uso de drogas nas universidades podem utilizar essas informações, a fim de desenvolver estratégias eficazes que considerem a percepção dos estudantes como um elemento crítico, no processo de experimentação e uso de drogas, por parte dos acadêmicos de instituições de ensino superior. Novos estudos serão importantes para replicar os resultados e, além disso, relacionar os programas desenvolvidos à correção das percepções errôneas por parte dos estudantes.
A principal limitação do estudo refere-se ao fato de que o perfil real do consumo de drogas ilícitas pode ser maior do que o resultado apresentado neste estudo, uma vez que muitos estudantes não respondem corretamente aos questionários. A maioria dos estudantes têm medo do preconceito, do estigma social e da justiça. Dessa forma, esses três fatores limitaram claramente a realização do estudo.

\section{Referências}

1. Lucas ACS, Parente RCP, Picanço NS, Conceição DA, Costa KRC, Magalhães IRS. Uso de psicotrópicos entre universitários da área da saúde da Universidade Federal do Amazonas, Brasil. Cad Saúde Pública. 2006;22(3):663-71. 2. Peuker AC, Fogaça J, Bizarro L. Expectativas e beber problemático entre universitários. Psicol Teoria Pesqui. 2006;22(2):193-200.

3. Secretaria Nacional de Políticas sobre Drogas, SENAD (BR). I Levantamento Nacional sobre Uso de Álcool, Tabaco e Outras Drogas entre Universitários das 27 capitais brasileiras. Brasília (DF): SEND; 2010.

4. Silva LVER, Malbergier A, Stempliuk VA, Andrade AG. Fatores associados ao consumo de álcool e drogas entre estudantes universitários. Rev Saúde Pública. 2006;40(2):280-8.

5. Pillon SC, O'Brien B, Piedra Chavez KA. The relationship between drug use and risk behaviors in Brazilian university students. Rev Lat-Am Enfermagem. 2005;13 Spec No:1169-76.

6. Pechansky F, Szobot CM, Scivoletto S. Uso de álcool entre adolescentes: conceitos, características epidemiológicas e fatores etiopatogênicos. Rev Bras Psiquiatr. 2004;26(1):14-7.

7. Carlini-Contrim B, Gazal-Carvalho C, Gouveira N. Comportamento de saúde entre jovens estudantes das redes públicas e privadas da área metropolitana do Estado de São Paulo. Rev Saúde Pública. 2004;34(6):636-45.

8. Murphy JG, Mc Devitt-Murphy ME, Barnett NP. Drink and be merry? Gender, life satisfaction, and alcohol consumption among college students. Psychol Addict Behav. 2005;19(2):184-91.

9. Polit DF, Beck CT, Hungler BP. Fundamentos de pesquisa em enfermagem: métodos, avaliação e utilização. 5 ed. Porto Alegre: Artmed; 2004.

10. Gil AC. Como Elaborar Projetos de Pesquisa. São Paulo: Atlas; 2006.

11. Oliveira HPJ, Brands B, Cunningham J, Strike C, Wright MGM. Percepção dos Estudantes Universitários sobre o Consumo de Drogas entre seus pares no Abc Paulista, São Paulo, Brasil. Rev. Latino-Am. Enfermagem. 2009;17(spec):871-7.

12. Tockus D, Gonçalves PS. Detecção do uso de drogas de abuso por estudantes de medicina. J Bras Psiquiatr. 2008;57(3):184-7.

13. Fiorini JE, Alves AL. Uso de drogas lícitas e ilícitas no meio universitário de Alfenas. Rev Univ Alfenas 1999;5:263-7.

14. Chiapetti N, Serbena CA. Uso de álcool, tabaco e 
drogas por estudantes da área de saúde de uma universidade de Curitiba. Psicol Reflexão e Crítica 2007;20(2):303-13.

15. Mardegan PS. Uso de substâncias psicoativas entre estudantes de enfermagem. J Bras Psiquiatr. 2007;56(4):260-6.

16. Picolotto E, Libardoni LFC, Migott AMB, Geib LTC. Prevalência e fatores associados com o consumo de substâncias psicoativas por acadêmicos de enfermagem da Universidade de Passo Fundo. Ciênc Saúde Colet. 2010;15(3):645-54.

17. Carlini EA, Carlini-Contrim B, Silva-Filho AR. Sugestões para Programas de Prevenção ao Abuso de Drogas no Brasil. São Paulo: Centro Brasileiro de Informações sobre Drogas Psicotrópicas (CEBRID). Departamento de Psicobiologia da Universidade Federal de São Paulo; 1990. 